\title{
Friedel oscillations in a Luttinger liquid with long-range interactions
}

\author{
Victoria I. Fernández $z^{1,2, *}$ and Carlos M. Naón ${ }^{1,2, \dagger}$ \\ ${ }^{1}$ Instituto de Física La Plata, Departamento de Física, Facultad de Ciencias Exactas, Universidad Nacional de La Plata, CC 67, \\ 1900 La Plata, Argentina \\ ${ }^{2}$ Consejo Nacional de Investigaciones Cientificas y Técnicas, 1900 La Plata, Buenos Aires, Argentina \\ (Received 5 October 2000; revised manuscript received 5 March 2001; published 8 June 2001)
}

\begin{abstract}
We introduce a path-integral approach that allows us to compute charge-density oscillations in a Luttinger liquid with impurities. We obtain an explicit expression for the envelope of Friedel oscillations in the presence of arbitrary electron-electron potentials. As examples, in order to illustrate the procedure, we show how to use our formula for contact and Coulomb potentials.
\end{abstract}

DOI: 10.1103/PhysRevB.64.033402

PACS number(s): 05.30.Fk, 71.10.Pm

In the last few years there has been much activity addressed to the study of condensed matter and statistical mechanics problems through field-theoretical methods. ${ }^{1}$ In particular, the physics of one-dimensional (1D) systems of strongly correlated particles has become a very interesting subject since one can take advantage of the simplicity of the models at hand and, at the same time, expect to make contact with experiments. For instance, the recently built quantum wire $^{2}$ is a good realization of a 1D electron gas. From the theoretical side, the simplest formulation of a $1 \mathrm{D}$ electronic system is given by the Tomonaga-Luttinger (TL) model, ${ }^{3}$ which has been successful in describing some qualitative features of a Luttinger liquid such as spin-charge separation and nonuniversal exponents in the decay law of correlation functions. ${ }^{4}$ There are, however, two crucial issues that are not considered in the original versions of this model: the presence of a nontrivial interaction between electrons and impurities $^{5}$ and the effect of long-range (LR) electronelectron interactions. ${ }^{6,7}$ As is well known, the former leads to the occurrence of Friedel oscillations in the charge-density profile, at least for Fermi liquids. ${ }^{8}$ On the other hand, as the dimensionality of a system decreases, charge screening effects become less important and the LR interaction between electrons is expected to play a central role in determining the properties of the system. In fact, from a theoretical point of view, the effects of LR interactions have been recently discussed in connection to several problems such as the Fermiedge singularity, ${ }^{9}$ the insulator-metal transition, ${ }^{10}$ and the role of the lattice through umklapp scattering and sizedependent effects. ${ }^{11}$ Thus, it is quite interesting to study the interplay between impurities and LR interactions by considering Friedel oscillations in a 1D system. Some time ago, Egger and Grabert ${ }^{12}$ analyzed this phenomenon. By combining the techniques of standard bosonization ${ }^{13}$ with the selfconsistent harmonic approximation ${ }^{14}$ and quantum Monte Carlo simulations, ${ }^{15}$ they were able to get explicit results for both weak and strong impurity scattering regimes. Later on, the authors of Ref. 16 used bosonization and a scattering description to get some exact results for the short-range case and for a special value of the coupling constant, equivalent to the so called "Toulouse point" in the anisotropic Kondo problem. More recently, the authors of Ref. 17 again used standard bosonization to address the same problem empha- sizing the equivalence between the TL model in the presence of a single nonmagnetic impurity and a boundary SineGordon model.

In this report, we present an alternative approach to this problem based on a path-integral bosonization technique previously developed in the context of nonlocal quantum field theories. ${ }^{18}$ This method seems to be specially adequate to consider LR interactions. Indeed, it has recently provided a straightforward derivation of the electronic Green's function in the presence of noncontact potentials. ${ }^{19}$ Then, our main purpose here is to show how to extend this formulation to the computation of Friedel oscillations.

We start from a modified nonlocal Thirring model $^{20}$ described by the following (Euclidean) Lagrangian density:

$$
\begin{aligned}
\mathcal{L}= & i \bar{\Psi}\left(b+\gamma_{0} k_{F}\right) \Psi+\int d^{2} y J_{\mu}(x) U_{(\mu)}(x, y) J_{\mu}(y)+\bar{\Psi} \mathbb{C} \Psi \\
& -M(x) \bar{\Psi} \Psi
\end{aligned}
$$

where $x=\left(\tau_{x}, \mathbf{x}\right)=\left(x_{0}, x_{1}\right)$, and $J_{\mu}=\bar{\Psi} \gamma_{\mu} \Psi$. The functions $U_{(\mu)}(x, y)$ are forward-scattering potentials. Setting $U_{(0)}$ $=U_{(1)}=-\delta^{2}(x-y)$, one gets the covariant and local version of the Thirring model usually studied in the context of $(1+1)$ quantum field theories $\left(\mathrm{QFT}^{\prime} \mathrm{s}\right)$.

On the other hand, the choice $U_{(0)}(x, y)$ $=U(|\mathbf{x}-\mathbf{y}|) \quad \delta\left(\tau_{x}-\tau_{y}\right)$ and $U_{(1)}(x, y)=0$, yields the simplest version of the Tomonaga-Luttinger (TL) model with an instantaneous distance-dependent potential and no currentcurrent fluctuations. The last two terms in Eq. (1) correspond to forward and backward electron-impurity scattering, respectively.

The main purpose of the present paper is to evaluate the vacuum expectation value (VEV) of the charge density:

$$
\langle\rho(x)\rangle=\left\langle\Psi^{\dagger} \Psi+e^{-2 i k_{F} \mathbf{x}} \Psi_{R}^{\dagger} \Psi_{L}+e^{2 i k_{F} \mathbf{x}} \Psi_{L}^{\dagger} \Psi_{R}\right\rangle,
$$

for an arbitrary electron-electron potential $U_{(\mu)}(x, y)$. Also, we will be especially interested in case the impurity terms are $C_{0}(x)=V \delta(\mathbf{x}-d)=M(x)$ and $C_{1}(x)=0$, where $V$ is a constant proportional to the impurity tunneling barrier situated at $\mathbf{x}=d$. Using a suitable representation of the functional delta and introducing an auxiliary vector field $A_{\mu}$ (see Ref. 18 for details), the partition function of the model under consideration reads 


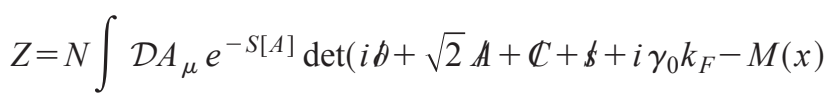

$$
\begin{aligned}
& \left.+s_{\mu} \epsilon_{\mu \nu} e^{2 i k_{F} \mathbf{x} \Gamma_{\nu}}\right),
\end{aligned}
$$

where $S[A]$ is the free quadratic action for $A_{\mu}, \Gamma_{0}=\mathbf{I}$ and $\Gamma_{1}=\gamma_{5}$. Equation (2) can be obtained by functional derivation of Eq. (3) with respect to the source $s_{0}$.

As it is known, the massivelike determinant in Eq. (3) cannot be exactly solved, even in the local case. However, we can take advantage of the fact that the vacuum to vacuum functional can be written in such a way that nonlocal terms are not present in the determinant. Therefore, the terms $\sqrt{2} \mathfrak{A}+\mathbb{C}+\mathfrak{b}+i \gamma_{0} k_{F}$ can be decoupled from fermions by performing chiral and gauge transformations in the fermionic path-integral measure. Indeed, decomposing $A_{\mu}(x)$ in longitudinal and transverse pieces

$$
\begin{aligned}
A_{\mu}(x)= & \epsilon_{\mu \nu} \partial_{\nu}\left(\Phi(x)-\frac{i k_{F} \mathbf{x}}{\sqrt{2}}\right)+\partial_{\mu} \eta(x) \\
& -\frac{1}{\sqrt{2}}\left[C_{\mu}(x)+s_{\mu}(x)\right],
\end{aligned}
$$

where $\Phi$ and $\eta$ are boson fields (to be associated to the normal modes of the system) and applying, as anticipated, functional bosonization techniques ${ }^{18}$ to express the fermionic determinant in terms of $\Phi$ and $\eta$, one finally obtains

$$
\begin{aligned}
Z= & N \int \mathcal{D} \overline{\bar{\chi}} \mathcal{D} \chi \mathcal{D} \Phi \mathcal{D} \eta \exp \left(-S_{b o s}\right) \exp \left(-S_{f e r}\right) \\
& \times \exp \left(-S\left[M, s_{\mu}\right]\right),
\end{aligned}
$$

where $S_{f e r}$ corresponds to free massless fermions ( $\chi$ and $\bar{\chi}$ ) and

$$
S\left[M, s_{\mu}\right]=\int d^{2} x \bar{\chi}\left[s_{\mu}(x) \epsilon_{\mu \nu} e^{2 i k_{F} \mathbf{x} \Gamma_{\nu}}-M(x)\right] e^{-2 g \gamma_{5} \Phi} \chi .
$$

Concerning $S_{b o s}$, it can be more briefly described in momentum space:

$$
\begin{aligned}
S_{b o s}= & \int \frac{d^{2} p}{(2 \pi)^{2}}\left[\hat{\Phi}(p) \hat{\eta}(p) \hat{C}_{\mu}^{\prime}(p)\right] \\
& \times\left(\begin{array}{ccc}
A(p) & \frac{C(p)}{2} & \frac{E(p)}{2} \\
\frac{C(p)}{2} & B(p) & \frac{F(p)}{2} \\
\frac{E(p)}{2} & \frac{F(p)}{2} & D(p)
\end{array}\right)\left(\begin{array}{c}
\hat{\Phi}(-p) \\
\hat{\eta}(-p) \\
\hat{C}_{\mu}^{\prime}(-p)
\end{array}\right) \\
& +\frac{i k_{F}}{2} \int \frac{d^{2} p}{(2 \pi)^{2}} \hat{U}_{(0)}^{-1}(p) \hat{C}_{(0)}^{\prime}(p) \delta^{2}(p),
\end{aligned}
$$

where we have defined $C_{\mu}^{\prime}=C_{\mu}+s_{\mu} \quad$ and $A(p), B(p), C(p), D(p), E(p)$, and $F(p)$ are potentialdependent functions (see Ref. 20 for more details).

At this point, we see that the generating functional can be formally expanded in powers of $\left(M-s_{\mu} \epsilon_{\mu \nu} e^{2 i k_{F} \mathbf{x} \Gamma_{\nu}}\right)$, in complete analogy with the usual procedure employed in the path-integral bosonization of $(1+1)$ massive QFT's. ${ }^{21,22} \mathrm{In}$ fact, the $x$ dependence of this perturbative parameter, together with the appearance of $C_{\mu}^{\prime}(x)$ in the bosonic action are two of the new features of the present computation. As long as these functions are well behaved, one can assume the existence of every term in the corresponding series.

From now on, we will specialize the computation to the case $s_{1}=0$. This allows us to define $M_{ \pm}(x)=M(x)$ $-s_{0}(x) e^{\mp 2 i k_{F} \mathbf{X}}$ and one can then perform the abovementioned expansion of $Z$ taking $M_{ \pm}(x)$ as perturbative parameters. As explained in Ref. 20, one can show that the same expansion can be obtained by starting from a purely bosonic nonlocal extension of the sine-Gordon model given by

$$
\begin{aligned}
\mathcal{L}^{\prime}= & \frac{1}{2}\left(\partial_{\mu} \varphi\right)^{2}+\frac{1}{2} \int d^{2} y \partial_{\mu} \varphi(x) d_{(\mu)}(x, y) \partial_{\mu} \varphi(y) \\
& +F_{\mu} \partial_{\mu} \varphi-\frac{1}{2 \beta^{2}}\left(\alpha_{+}(x) e^{i \beta \varphi(x)}+\alpha_{-}(x) e^{-i \beta \varphi(x)}\right),
\end{aligned}
$$

where $F_{\mu}(x)$ represents a couple of classical functions to be related to the $C_{\mu}^{\prime}$ 's and $d_{(\mu)}(x, y)$ are two bilocal functions that will be associated to the electron-electron potentials (a similar nonlocality in the kinetic term was considered in the study of the influence of LR correlations in the metalinsulator transition $\left.{ }^{23}\right), \beta$ is a constant and $\alpha_{ \pm}(x)$ are functions that can be considered as extensions of the parameter $\alpha_{0}$ used by Coleman. ${ }^{24}$ Indeed, for $d_{(\mu)}=0=F_{\mu}$ and $\alpha_{+}$ $=\alpha_{-}=\alpha_{0}=$ constant , the model above coincides with the usual sine-Gordon model. In the present approach, the quantities $\alpha_{ \pm}(x)$ are related to $M_{ \pm}(x)$, which are in turn connected to the strength of the scatterer. Let us stress that in our formulation, it is straightforward to consider a nonpointlike impurity $\left[\alpha_{ \pm}(x) \neq V \delta(\mathbf{x}-d)\right]$. However, in order to illustrate our method, in this report we will consider the usual case of a completely localized impurity. For this particular case, Eq. (8) contains the same terms that can be derived from standard bosonization (see, for instance, Ref. 12).

Now, going to momentum space and employing standard procedures to evaluate each $\mathrm{VEV}$, the partition function $Z^{\prime}$ corresponding to this generalized sine-Gordon model coincides with $Z$ provided that the following three relations hold:

$$
\begin{gathered}
\frac{2 \pi}{\frac{2}{\pi}\left[p_{0}^{2} \hat{U}_{(1)}(p)+p_{1}^{2} \hat{U}_{(0)}(p)\right]+p^{2}} \\
=\frac{\beta^{2}}{2\left[p^{2}+\hat{d}_{(0)}(p) p_{0}^{2}+\hat{d}_{(1)}(p) p_{1}^{2}\right]},
\end{gathered}
$$




$$
\begin{gathered}
\frac{\alpha_{ \pm}(x)}{\beta^{2}}=M_{ \pm}(x)=M(x)-s_{0}(x) e^{\mp 2 i k_{F} \mathbf{x}}, \\
\frac{2 i \hat{C}_{\mu}^{\prime}(-p) \epsilon_{\mu \nu} p_{\nu}}{\frac{2}{\pi}\left[p_{0}^{2} \hat{U}_{(1)}(p)+p_{1}^{2} \hat{U}_{(0)}(p)\right]+p^{2}}=\frac{\beta \hat{F}_{\mu}(-p) p_{\mu}}{p^{2}+\hat{d}_{(0)} p_{0}^{2}+\hat{d}_{(1)} p_{1}^{2}} .
\end{gathered}
$$

Therefore, we have obtained an equivalence between the partition functions $Z$ and $Z^{\prime}$ corresponding to the nonlocal Thirring and sine-Gordon models with extra interactions defined above. This means that we can use $Z^{\prime}$ together with the above conditions in order to compute the charge-density in the Luttinger liquid in the presence of impurities. Indeed, as a result of this bosonization technique, we can evaluate $\langle\rho(x)\rangle$ through functional derivation of $Z^{\prime}$ instead of $Z$. In so doing we obtain:

$$
\langle\rho(x)\rangle=\left\langle\frac{i}{\sqrt{\pi}} \partial_{\mathbf{x}} \varphi(x)+\cos \left[2 \sqrt{\pi} \varphi(x)-2 k_{F} \mathbf{x}\right]\right\rangle
$$

where the VEV is taken with respect to the Lagrangian density $\mathcal{L}^{\prime}\left[s_{0}=0\right]$ obtained from Eq. (8) after using Eqs. (9), (10), and (11) and setting $s_{0}=0$. Note that we have also set $\beta=2 \sqrt{\pi}$.

Let us remark that there is an additional contribution to Eq. (12), coming from the functional derivative of the normalization constant $N^{\prime}\left[C_{\mu}^{\prime}\right]$ with respect to $s_{\mu}$. Since this quantity is a constant, its only effect is to shift the background value of the charge density. For this reason we have just disregarded it.

Now we return to our main goal, that is to use the pathintegral framework depicted above in order to obtain an explicit formula for the charge density in a Luttinger liquid with arbitrary electron-electron and electron-impurity interactions. When one imposes these conditions in $\mathcal{L}^{\prime}\left[s_{0}=0\right]$, one gets a Lagrangian density that has an undefined parity as a function of $\varphi$. However it is much simpler to work with an even Lagrangian since, in this case, all VEV's of odd functions of $\varphi$ will vanish. It is easy to see that the translation $\varphi(x) \rightarrow \varphi(x)+f(x)$ yields an even Lagrangian $\mathcal{L}_{\text {even }}^{\prime}$ provided that the classical function $f(x)$ is $\tau_{x}$ independent and its gradient satisfies:

$$
\partial_{\mathbf{x}} f(x)+\frac{2}{\pi} \int d \mathbf{y} U(\mathbf{x}-\mathbf{y}) \partial_{\mathbf{y}} f\left(\tau_{x}, \mathbf{y}\right)+\frac{i}{\sqrt{\pi}} V \delta(\mathbf{x}-d)=0 .
$$

We then get

$$
\begin{aligned}
\langle\rho(x)\rangle= & \frac{i}{\sqrt{\pi}} \partial_{\mathbf{x}} f+\cos \left[\sqrt{4 \pi} f(\mathbf{x})-2 k_{F} \mathbf{x}\right] \\
& \times\langle\cos [\sqrt{4 \pi} \varphi(x)]\rangle_{\mathcal{L}_{\text {even }}^{\prime}},
\end{aligned}
$$

where $\cos \left[\sqrt{4 \pi} f(\mathbf{x})-2 k_{F} \mathbf{x}\right]$ is called the Friedel oscillation and $A(x)=\langle\cos [\sqrt{4 \pi} \varphi(x)]\rangle_{\mathcal{L}_{\text {even }}^{\prime}}$ is the corresponding envelope.
Let us point out that Eq. (13) has been previously found in Ref. 17. As shown by these authors, in the short-range case it has the solution $f=$ constant $\propto U$ whose only effect is to add a constant phase in the cosine term associated to the Friedel oscillation. A nontrivial phenomenon takes place for LR potentials, since the cosine ceases to be a periodic function. This nonperiodicity effect, although weak at large distances, could eventually be observed in carbon nanotubes. ${ }^{25}$

From now on we shall focus our attention on the computation of the envelope of the oscillation. Since $\mathcal{L}_{\text {even }}^{\prime}$ is not exactly solvable, we shall employ the well-known selfconsistent harmonic approximation, ${ }^{14}$ which amounts to replacing $\mathcal{L}_{\text {even }}^{\prime}$ by

$$
\begin{aligned}
\mathcal{L}_{S C H A}= & \frac{1}{2}\left(\partial_{\mu} \varphi\right)^{2}+\frac{1}{\pi} \int d \mathbf{y} \partial_{\mathbf{x}} \varphi\left(\tau_{x}, \mathbf{x}\right) U(\mathbf{x}-\mathbf{y}) \partial_{\mathbf{y}} \varphi\left(\tau_{x}, \mathbf{y}\right) \\
& +\frac{m(V) \delta(\mathbf{x}-d)}{2} \varphi^{2},
\end{aligned}
$$

where $m(V)$ is a constant, related to the impurity strength, to be variationally determined. The precise relationship between $m(V)$ and $V$ was obtained in Ref. 12. For instance, in the strong-scattering limit, when $V$ is much larger than a certain bandwidth, one has simply $m=V$ (See also, Ref. 17).

Let us now consider the computation of $A(x)$ using this approximation. Performing a translation in the field $\varphi(x)$ $\rightarrow \varphi(x)+a(x)$, with $a(x)$ a classical function, we find $A(x)=\exp i \sqrt{\pi} a(x)$.

Going to momentum space we see that the Fourier transform of $a(x)$ satisfies an integral equation whose solution is

$$
\hat{a}(p)=\frac{2 i \sqrt{\pi}}{p^{2}+p_{1}^{2} \frac{2 U\left(p_{1}\right)}{\pi}} e^{-i p_{\mu} x_{\mu}}\left(1-\frac{m e^{i p_{1} r} I\left(p_{0}, r\right)}{\pi+m I\left(p_{0}, 0\right)}\right),
$$

with

$$
I\left(p_{0}, r\right)=\int_{0}^{\infty} d q_{1} \frac{\cos \left(q_{1} r\right)}{p_{0}^{2}+q_{1}^{2}\left(1+\frac{2 U\left(q_{1}\right)}{\pi}\right)},
$$

where we defined $r=|\mathbf{x}-d|$.

The envelope of the Friedel oscillation then reads

$$
A(r)=\exp -\frac{1}{\pi} \int_{-\infty}^{\infty} d p_{0}\left(I\left(p_{0}, 0\right)-\frac{m I^{2}\left(p_{0}, r\right)}{\pi+m I\left(p_{0}, 0\right)}\right),
$$

which is our main formal result. Indeed, formulas (17) and (18) give an analytical expression (exact within the Gaussian approximation) for $A(r)$ as a function of both the electronelectron potential and the variational parameter $m(V)$. Since the self-consistent harmonic approximation seems to fail for weak impurity strength, due to the neglect of interwell tunneling, ${ }^{12}$ we restrict our analysis to the strong impurity regime. We also consider a large distance approximation of Eq. (17) which consists of inserting $1 / r$ as infrared cutoff. Let us call $I_{r}\left(p_{0}, r\right)$ the integral (17) regulated in this way. 
We will examine, as examples, two specific short-range and Coulomb potentials. This, in turn will allow us to illustrate how to use our general formula (2) for other cases. Moreover, since these problems were previously considered in Refs. 12 and 17 by using standard (operational) bosonization, our computation will give an independent confirmation by means of a different approach. First of all, we note that it is convenient to split out the two terms of the exponential factor on Eq. (18), such that $A(r)=\exp (T+W)(r)$.

For the simple contact potential $U\left(q_{1}\right)=U=$ constant, we get

$$
T(r)=\ln (\Lambda r)^{-g},
$$

and

$$
W(r)=g \exp \left(m g^{2} r\right) E_{i}\left(-m g^{2} r\right)-g \exp (2 g) E_{i}(-2 g),
$$

where $\Lambda$ is an ultraviolet cutoff. We have also introduced the interaction constant $g=(1+2 U / \pi)^{-1 / 2}$. Taking into account the asymptotic behavior of the exponential integral function $E_{i}$ for $m g^{2} r \gg 1$, one obtains

$$
A(r)=\mathcal{C}(g, \Lambda)(2 g r)^{-g} \exp \left(-\frac{1}{m g r}\right),
$$

which coincides with Refs. 12 and 17 under the same regime.

In the Coulombian case, one has $U(|\mathbf{x}|)=U / \sqrt{|\mathbf{x}|^{2}+b^{2}}$, whose Fourier transform is $U\left(q_{1}\right)=2 U K_{0}\left(b q_{1}\right)$, where $b$ plays the role of a lattice spacing. Inserting this expression in $T$ and $W$, and considering the same regime as before we find that $W$ vanishes and
$T(r)=-\frac{\pi}{2 U}\left(\sqrt{1-\frac{4 U}{\pi} \ln \frac{b}{2 r}}-\sqrt{1-\frac{4 U}{\pi} \ln \frac{b \Lambda}{2}}\right)$,

which yields

$$
A(r)=\mathcal{C}^{\prime}(g, b, \Lambda) \exp \left(-\sqrt{\frac{\pi}{U} \ln \frac{r}{b}}\right) .
$$

Again, this behavior is equal to the one previously found in Refs. 12 and 17 .

In summary, we have described an alternative bosonization approach to the computation of charge-density fluctuations. This technique, previously originated in the context of QFT's, parallels, in the path-integral framework, the operational schemes usually employed in condensed-matter applications. In particular, we have computed the envelope of Friedel oscillations in a simple version of the TL model with a nonmagnetic impurity. By combining that bosonization procedure and the self-consistent harmonic approximation, we were able to express the envelope of the oscillations as a function of the electron-electron interaction [see Eqs. (17) and (18)]. Finally, as a consistency check of this formal result, and in order to illustrate our method, we considered the long-distance regime for contact interactions and Coulomb potentials. Our results are in agreement with Refs. 12 and 17.

This work was partially supported by the Consejo Nacional de Investigaciones Científicas y Técnicas (CONICET), Argentina. We are grateful to Aníbal Iucci for useful discussions. We thank Kang Li for calling our attention to Ref. 17.
*Email address: victoria@venus.fisica.unlp.edu.ar

†Email address: naon@venus.fisica.unlp.edu.ar

${ }^{1}$ Eduardo Franklin, Field Theories of Condensed Matter Physics, Frontiers in Physics (Addison-Wesley, Reading, MA, 1991).

${ }^{2}$ M. A. Kastner, Rev. Mod. Phys. 64, 849 (1992); S. Tarucha et al., Phys. Rev. B 47, 4064 (1993).

${ }^{3}$ S. Tomonaga, Prog. Theor. Phys. 5, 544 (1950); J. Luttinger, J. Math. Phys. 4, 1154 (1963); E. Lieb and D. Mattis, ibid. 6, 304 (1965).

${ }^{4}$ J. Voit, Rep. Progr. Phys. 58, 977 (1995).

${ }^{5}$ C. L. Kane and M. P. A. Fisher, Phys. Rev. B 46, 15233 (1992).

${ }^{6}$ H. J. Schulz, Phys. Rev. Lett. 71, 1864 (1993).

${ }^{7}$ H. Otani and T. Ogawa, Phys. Rev. B 54, 4540 (1996); D. Poilblanc et al., ibid. 56, R1645 (1997).

${ }^{8}$ J. Friedel, Nuovo Cimento Suppl. 7, 287 (1958); I. Tutto and A. Zawadowsky, Phys. Rev. B 32, 2449 (1985).

${ }^{9}$ H. Otani and T. Ogawa, Phys. Rev. B 54, 4540 (1996).

${ }^{10}$ D. Poilblanc et al., Phys. Rev. B 56, R1645 (1997).

${ }^{11}$ G. Fano et al., Phys. Rev. B 60, 15654 (1999); S. Capponi et al., ibid. 61, 13410 (2000).

${ }^{12}$ R. Egger and H. Grabert, Phys. Rev. Lett. 75, 3505 (1995).

${ }^{13}$ V. J. Emery, in Highly Conducting One-dimensional Solids, edited by J. T. Devreese et al. (Plenum, New York, 1979), p. 327; M. Stone, Bosonization (World Scientific, Singapore, 1994); R. Shankar, in Low Dimensional Quantum Field Theories for Con- densed Matter Physicists, edited by Lu Yu, S. Lundqvist, and G. Morandi (World Scientific, Singapore, 1995).

${ }^{14}$ Y. Saito, Z. Phys. B 32, 75 (1978); M. Stevenson, Phys. Rev. D 32, 1389 (1985); M. P. A. Fisher and W. Zwerger, Phys. Rev. B 32, 6190 (1985); A. O. Gogolin, Phys. Rev. Lett. 71, 2995 (1993); B. W. Xu and Y. M. Zhang, J. Phys. A 29, 7349 (1996).

${ }^{15}$ D. M. Ceperley and M. H. Kalos, in Monte Carlo Methods in Condensed Matter Physics, edited by K. Binder, Topics in Current Physics Vol. 7 (Springer, Heidelberg, 1979); Quantum Monte Carlo Methods in Physics and Chemistry, Vol. 525 of NATO Advanced Studies Institute, Series C: Mathematical and Physical Sciences, edited by M. P. Nightingale and C. J. Umrigar (Kluwer, Academic Publishers, Dordrecht, 1998).

${ }^{16}$ A. Leclair et al., Phys. Rev. B 54, 13597 (1996).

${ }^{17}$ Q. Yuan et al., Phys. Rev. B 58, 1084 (1998).

${ }^{18}$ C. Naón et al., Nucl. Phys. B 435, 567 (1995); ibid. 485, 665

(1997); M. Manías et al., ibid. 525, 721 (1998).

${ }^{19}$ A. Iucci and C. Naón, Phys. Rev. B 61, 15530 (2000).

${ }^{20}$ V. Fernández et al., Phys. Lett. B 452, 98 (1999).

${ }^{21}$ C. Naón, Phys. Rev. D 31, 2035 (1985).

${ }^{22}$ Kang Li and Carlos Naón, J. Phys. A 31, 7929 (1998).

${ }^{23}$ I. V. Krive et al., Phys. Rev. B 52, 10865 (1995).

${ }^{24}$ S. Coleman, Phys. Rev. D 11, 2088 (1975).

${ }^{25}$ S. Tans et al., Nature (London) 386, 474 (1997); R. Egger and A. O. Gogolin, Phys. Rev. Lett. 79, 5082 (1997). 